\title{
]jfis
}

\section{Neurological Measurement of Human Trust in Automation Using Electroencephalogram}

\author{
Seeung $\mathrm{Oh}^{1}$, Younho Seong ${ }^{2}$, Sun $\mathrm{Yi}^{3}$, and Sangsung Park \\ ${ }^{1}$ Department of Applied Engineering Technology at North Carolina Agricultural and Technical State \\ University, Greensboro, USA \\ ${ }^{2}$ Industrial and systems engineering with North Carolina A\&T State University, Greensboro, NC, \\ USA.Greensboro, NC, USA \\ ${ }^{3}$ Mechanical Engineering at North Carolina A\&T State University, USA \\ ${ }^{4}$ CheongJu University, Cheongju, Korea
}

\begin{abstract}
In modern society, automation is sufficiently complex to conduct advanced tasks. The role of the human operator in controlling a complex automation is crucial for avoiding failures, reducing risk, and preventing unpredictable situations. Measuring the level of trust of human operators is vital in predicting their acceptance and reliance on automation. In this study, an electroencephalogram (EEG) is used to identify specific brainwaves under trusted and mistrusted cases of automation. A power spectrum analysis was used for a brainwave analysis. The results indicate that the power of the alpha and beta waves is stronger for a trusted situation, whereas the power of gamma waves was stronger for a mistrusted situation. When the level of human trust in automation increases, the use of automatic control increases. Therefore, the findings of this research will contribute to utilizing a neurological technology to measure the level of trust of the human operator, which can affect the decision-making and the overall performance of automation used in industries.
\end{abstract}

Keywords: Trust, Mistrust, Automation, Electroencephalogram (EEG), Power spectrum

\section{Introduction}

Trust plays a crucial role in contributing to cognitive complexity and increased uncertainty in sophisticated automation [1]. Muir [2] claimed that trust is developed by "the human's ability to estimate the predictability of the machine's behaviors," which is an idea adopted in this study. Several researchers found that trust is a vital factor that affects the misuse and disuse of automation [1,3]. Measuring the level of trust of a human operator in automation is crucial in predicting their strategies when using automation. However, trust is a multidimensional construct influenced by various factors, such as motives, intentions, and actions [4]; therefore, measuring trust is a challenge. How can we effectively measure human trust within the use of automation? As a method for measuring trust, questionnaires are mostly used to investigate trust in interpersonal relationships [5] and have been used with an empirical approach [6], and with a semi-automatic simulation of human and automation [7]. The questionnaires utilized in these studies are based on a theoretical or empirical approach, which might vary based on the researcher's theoretical orientation or particular simulation model. Neurological measures can be a direct and objective method to measure human trust. Therefore, this research used both a 
neurological measure as a quantitative method to measure human trust and a questionnaire as a qualitative method to examine the responses of human trust in automation.

Some studies have investigated the relationship between social constructs (i.e., trust) and neural or physiological evidence. Previous studies $[8,9]$ have utilized fMRI and efMRI to analyze brain regions with stimuli associated with trust and distrust. Other studies have used an electroencephalogram (EEG) to examine factors that may affect human trust and decision-making $[10,11]$. However, previous studies have not fully investigated specific brainwaves within the situations of trust and mistrust in automation.

This research aims to identify specific brainwaves in situations of trust and mistrust in automation using an EEG. An EEG is selected because of its benefits over other neurological technologies. An EEG can be an appropriate method for measuring the active neurological responses to external stimuli involving trust and mistrust in real-time [12], and thus is useful for experiments with a wide range of cognitive tasks and real-world applications in the future. The EEG records signals from the scalp and therefore has a few limitations, namely, sensitivity to various noises other than the external stimuli. Acknowledging these limitations, filters were applied prior to the analysis, such as the marking and removal of artifacts.

The results of this study can contribute to the design of automated systems requiring the trust of human operators to achieve the maximum performance and safety. By detecting a human operator's trust regarding automation, designers can improve automated systems by developing a user-friendly interface and effective training approach that can decrease the workload and increase the level of trust. Further, this study can be a means of monitoring the psychological state of human operators. This EEG method can be used to monitor a human operator and detect the operator's trust in an automated system. If a human operator mistrusts the automation, operator training or improvements to the automated system are ways to regain the operator's trust. If the human operator is unstable and unfocused owing to extreme stress and anxiety, the recommendation is to apply full automation rather than manual operation, particularly during an emergency.

\section{Methods}

\subsection{Participants}

A total of 28 volunteers participated in this experiment. The participants were undergraduate and graduate students recruited at North Carolina AandT State University. There were 14 male and 14 female participants. Each subject was over 18 years old with normal or corrected-to-normal vision, free of current or past neurological and psychiatric disorders, and able to read and understand English writing. There was no preference for right- or left-handed users, but all participants had the ability to use a keyboard and a mouse with their hands. The subjects read and signed an informed consent form prior to the experiment. After the participants were seated, detailed instructions were given for each experiment.

\subsection{EEG Recording}

The EEG data were recorded using a g.HIamp (256-multichannel amplifier), g.GAMMAsys (active electrode system with g. GAMMAcap), and g.Recorder (brain signal recording software) developed by g.tec, a medical engineering company. The subject's head was fitted with a cap of electrodes (g.GAMMAcap), and 20 electrodes selected according to the international 10-20 system of electrode placement (see Figure 1) were filled with an abrasive electrolyte gel using a syringe (see Figure 2). The subject was seated in front of a computer screen, which shows simulated driving, with a keyboard and a mouse in an isolated and quiet room.

Before each experiment began, the brainwaves of the participants were recorded to identify artifacts to mark and remove later, such as eye blinking, jaw clenching, and muscle movement. Then, as baseline data without any stimuli, their brainwaves were recorded when they were relaxed. During this time, they were instructed not to move or talk, and to watch a blank monitor for $1 \mathrm{~min}$. They were also asked not to talk or blink their eyes (if possible) to reduce artifacts from being recorded in their brainwaves. The settings used to record the brainwaves were as follows: the sampling frequency was set at $256 \mathrm{~Hz}$, the high-frequency filter was set at $60 \mathrm{~Hz}$, and the lowfrequency filter was set at $0.1 \mathrm{~Hz}$. During this EEG experiment, only the alpha $(8-13 \mathrm{~Hz})$, beta $(13-30 \mathrm{~Hz})$, and gamma (30-60 $\mathrm{Hz}$ ) waves, which are brainwaves related to decision-making under situations of trust and mistrust, were analyzed when the participants were asked to choose between manual or automatic control of a simulated driving game. Delta $(0.2-4 \mathrm{~Hz})$ and theta (4-8 Hz) waves occur when people are asleep, and were therefore not analyzed in this experiment. This experiment met the conditions of IRB 17-0159. 
Table 1. Experiment design

\begin{tabular}{cccccccccccccc}
\hline Trials & Training & Break & $\mathbf{1}$ & $\mathbf{2}$ & $\mathbf{3}$ & $\mathbf{3}$ & $\mathbf{4}$ & $\mathbf{5}$ & $\mathbf{6}$ & $\mathbf{7}$ & $\mathbf{8}$ & $\mathbf{9}$ & $\mathbf{1 0}$ \\
\hline $\begin{array}{c}\text { Number of cars avoided } \\
\text { before an accident }\end{array}$ & 25 & & 25 & 25 & 25 & 25 & 25 & 25 & 8 & 5 & 4 & 9 & 8 \\
$\begin{array}{c}\text { Auto control } \\
\text { performance (\%) }\end{array}$ & 100 & 100 & 100 & 100 & 100 & 100 & 100 & 32 & 20 & 16 & 36 & 32 \\
\hline
\end{tabular}

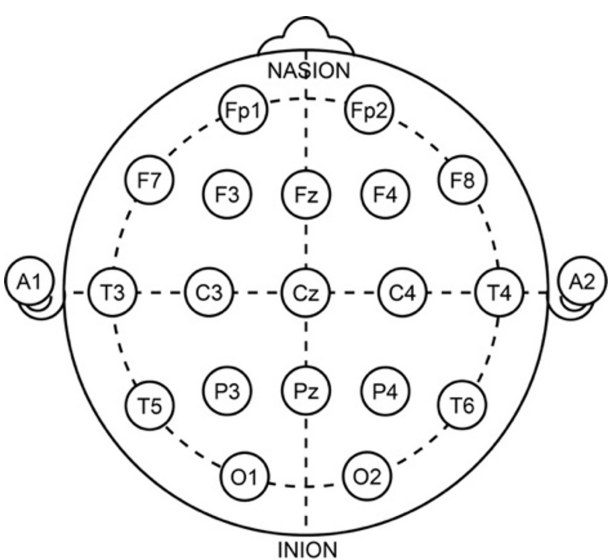

Figure 1. International 10-20 system for electrode placement [13].

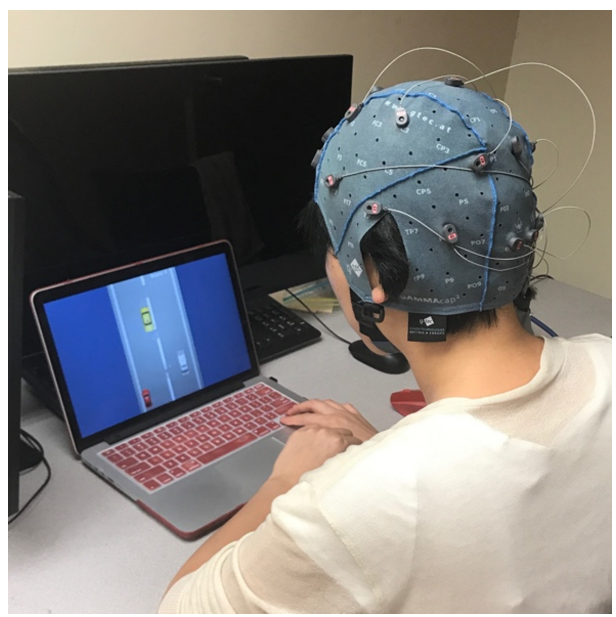

Figure 2. Example of EEG experiment.

\subsection{Experiment}

An experiment was designed to measure the neurological activities during the decision-making between automatic or manual control and to record the participants' use of such control. All 28 participants participated individually in six training sessions (three training sessions for automatic control and three for manual control), 10 simulated driving trials (see Table 1), and a survey to evaluate the level of trust between people and automation on a 7-point scale (see Appendix). During the training sessions, the participants practiced three trainings with automatic control (with a $100 \%$ performance) and three trainings with manual control (average performance of 50\%-60\%). This experiment was designed to allow the participants to choose automatic control to achieve a much better performance than manual control, and to evoke trust during the first five trials and mistrust for the remaining five trials. Mistrust is a strong and negative emotion that affects other emotions, and thus cannot be a counter-balance design. Trials 1-5 were designed to develop trust in automatic control by applying $100 \%$ automation, whereas trials 6-10 were designed to evoke mistrust in automation by applying a low performance of under $36 \%$, which is lower than the average performance under manual control (average of 50\%-60\%).

The performance was measured based on the number of cars avoided before an accident occurred. Successfully avoiding more than 25 cars resulted in a $100 \%$ performance rate, whereas avoiding only 1 car resulted in a $4 \%$ performance rate. At the beginning of each trial, the participant was required to choose either automatic or manual control to achieve a high performance rate. After choosing the type of control, the user could not change during the driving simulation. During each trial, the screen displayed the performance rate with a score (see Figure 3 ), and thus the previous performance affected the following decision of the participant. The brainwaves of each participants were recorded during each trial, including the decision-making process (before the simulation) and simulated driving period (driving). However, only brainwaves associated with decisionmaking (automatic or manual control) at the beginning of each trial were analyzed. In other words, this EEG experiment focuses only on the moment of the decision-making (thinking), not the simulated driving (action).

\subsection{EEG Analysis}

For analysis, the power spectral density (PSD) was utilized as a reliable and fast method [14] for defining specific brainwaves (i.e., alpha, beta, and gamma waves), which were an- 

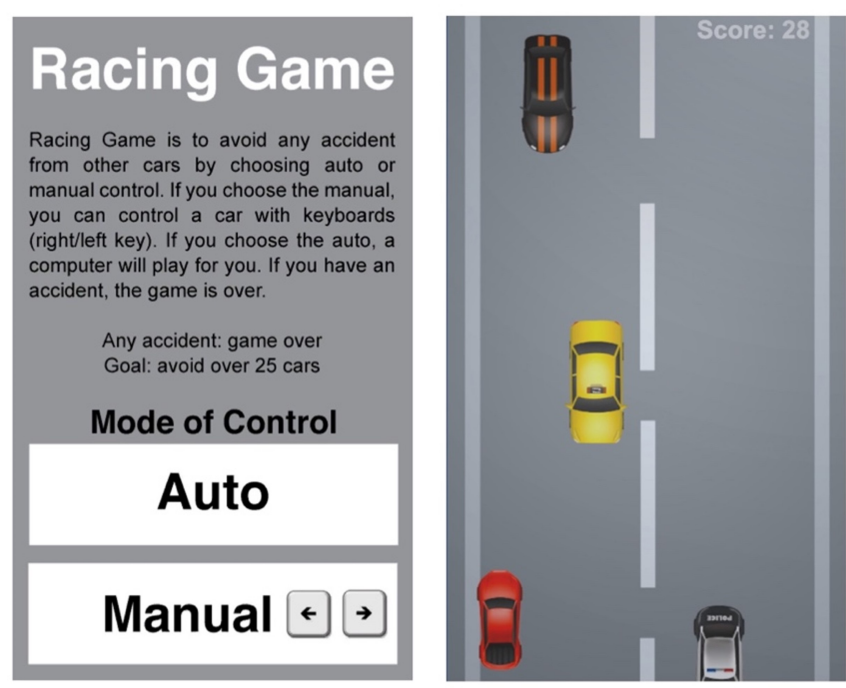

Figure 3. Example of simulated driving.

alyzed within the frequency domain to evaluate the state of trust and mistrust. A g.Recorder (brain signal recording software) recorded the raw EEG data, and marked artifacts were removed from the data using filters prior to the analysis. The processed EEG data were analyzed using a power spectrum analysis through the EMSE Suite Data Editor (EEG data editing software) developed by Cortech Solutions. Only alpha waves $(8-13 \mathrm{~Hz})$, beta waves $(13-30 \mathrm{~Hz})$, and gamma waves $(30-60 \mathrm{~Hz})$, which are related to decision-making, were analyzed during this experiment.

\section{Results}

\subsection{Rate of Automatic Control and the Trust Level}

After 28 participants completed 10 trials, they completed a survey to evaluate the level of trust between manual use and automation on a 7-point scale. The level of trust can be defined using a subjective trust questionnaire applying a trust scale [6, 7]. This survey follows a rating scale ranging from 1 ("not at all," which is close to mistrust) to 7 ("extremely," which is close to trust) [6].

For trials 1-6, the level of trust increased from 57\% to $94 \%$, whereas using the automatic control increased from 79\%-96\% (see Figure 4). By contrast, a 32\% error rate in the automatic control performance occurred in Trial 6, and trial 7 showed a substantial decrease in the level of trust from $94 \%$ to $30 \%$, as well as a decrease in automatic control from $96 \%$ to $21 \%$ (see Figure 4). These results imply that when the trust level in automation increases, the use of automatic control will increase.

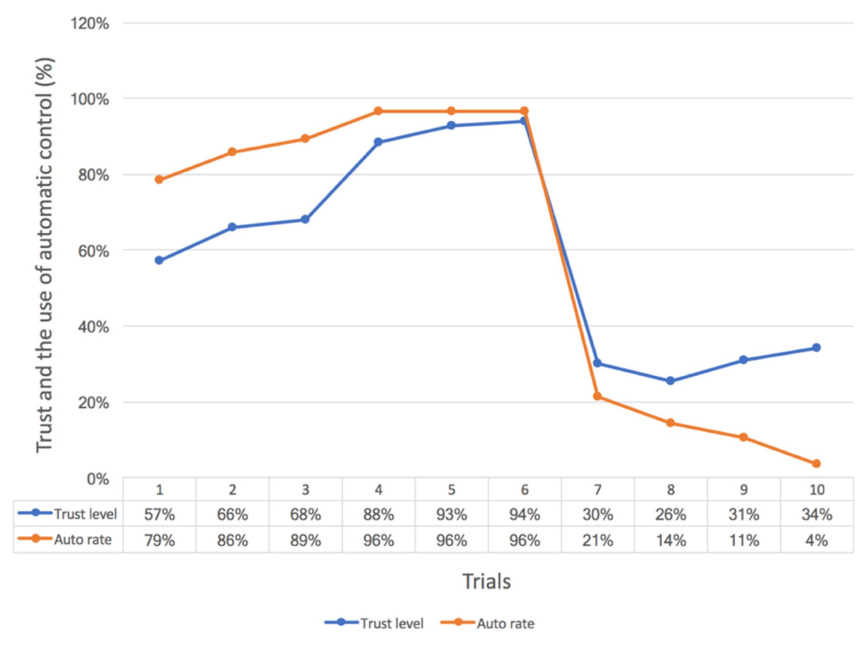

Figure 4. Comparison of trust level and use of automatic control.

Trust is a multidimensional construct influenced by various factors, and thus it is important to find which trial evokes trust or mistrust based on the trust level. It is important to understand that the previous performance affected the following decision. For example, the low performance (32\%) of automation of Trial 6 affected the decision of trial 7. Trial 5 shows $93 \%$ trust and trial 6 shows $94 \%$ trust, both of which are high levels. The brainwaves of trials 5 and 6 were analyzed to determine the state of trust. Trial 7 showed a $30 \%$ trust level, and trial 8 showed $26 \%$, which are low and indicate mistrust. The brainwaves of trials 7 and 8 were analyzed in terms a state of mistrust.

\subsection{Comparison of Intraindividual Differences}

The individual variability for the brainwaves is high owing to individual characteristics, and thus the analysis of this paper focuses on the intraindividual differences, showing the individual difference based on a baseline (standard) and the two stimuli of trust and mistrust applied in this study. This study focused on the changes in how states of trust and mistrust affect individual brainwaves. It did not compare the female and male results yet, but they can be investigated in the future.

Under a state of trust, the power of the alpha waves of 27 of the 28 participants highly increased, and the power of 1 participant (P16) decreased; under a state of mistrust, however, the power of the alpha waves of 26 of the 28 participants decreased slightly, and the power of two participants (P5 and P24) increased (see Figure 5). Under a state of trust, the power of the beta waves for all 28 participants increased considerably, whereas under a state of mistrust, the power of the beta waves for 26 of the 28 participants increased slightly, and the power 


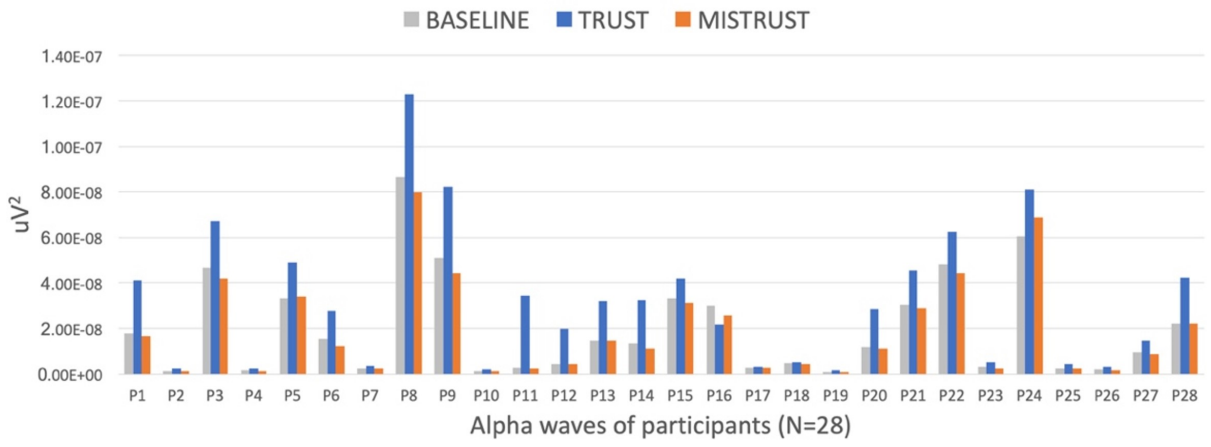

(a)

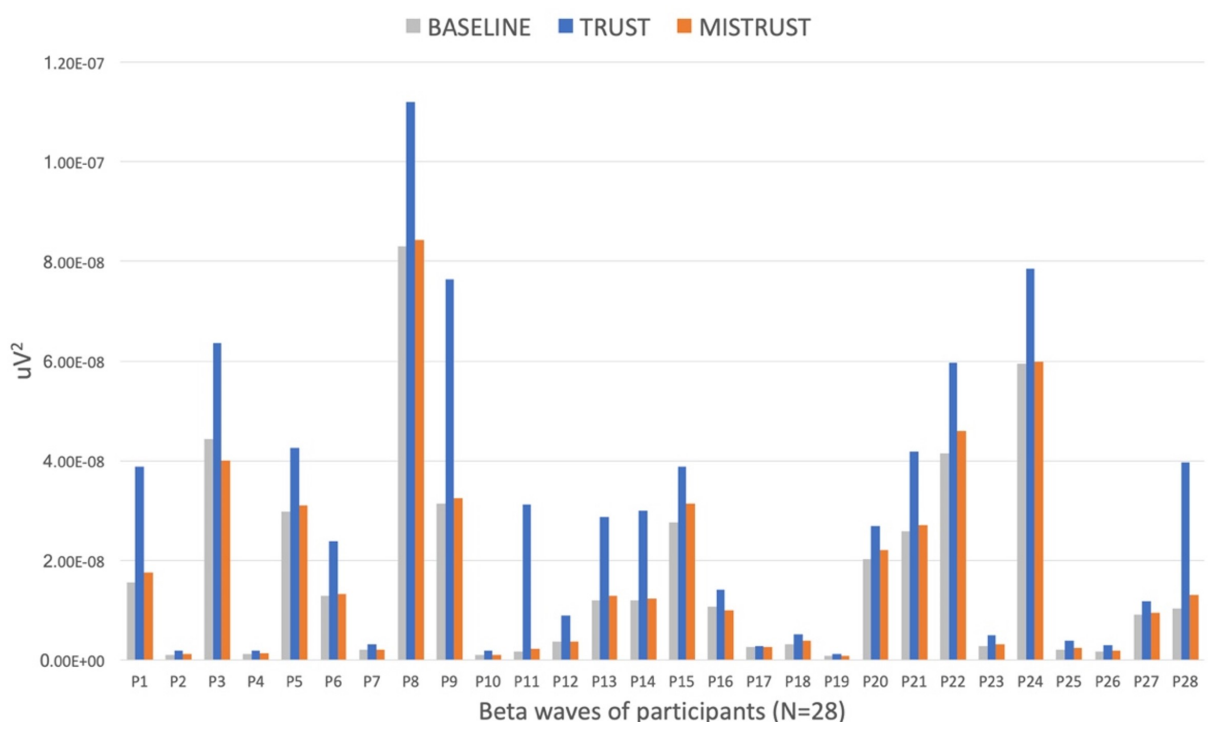

(b)

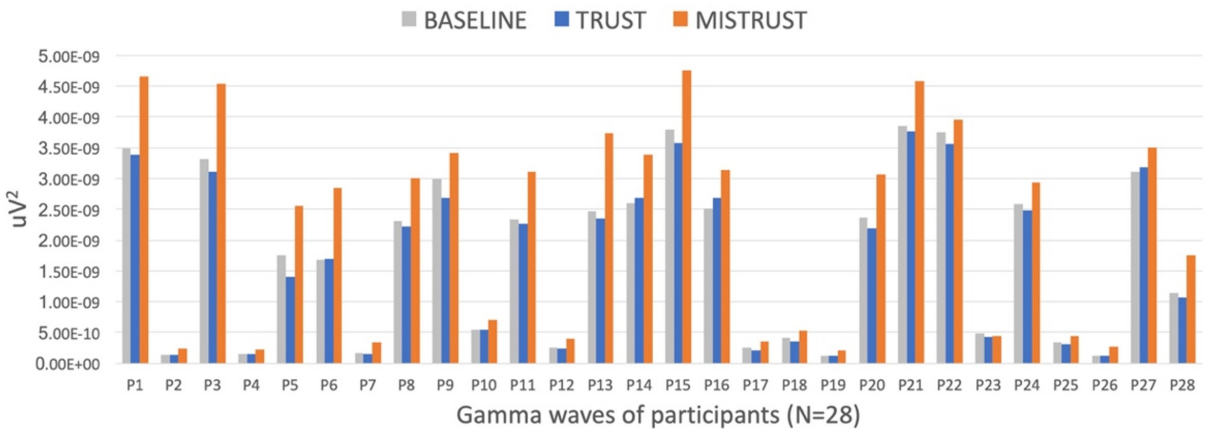

(c)

Figure 5. (a) Comparisons of the intraindividual differences in the alpha waves, (b) comparisons of the intraindividual differences in the beta waves(b), and (c) Comparisons of the intraindividual differences in the gamma waves.

of 2 participants (P3 and P16) decreased (see b in Figure 5). Under a state of trust, the power of the gamma waves decreased slightly for 22 of the 28 participants, but increased for 5 of the participants (P6, P14, P16, P26, and P27) and did not change for 1 participant (P4); under a state of mistrust, however, the power of the gamma waves increased significantly for the 27 of the 28 participants, but not for 1 participant (P23) (see c in Figure 5). There was 1 subject (P16) who chose more manual control than the other subjects, regardless of the performance of the automation. Under a state of trust, the alpha and beta waves 
of the subject (P16) decreased and were different from those of the other participants. Under a state of mistrust, the gamma waves of the subject (P16) increased, similar to that of the other participants. Some people have overconfidence in their abilities or a strong mistrust in machines, and therefore further research is needed in the future.

\subsection{Comparison of Trust and Mistrust Differences}

According to the results of the intraindividual differences, the alpha and beta waves are associated with a state of trust, whereas the gamma waves are associated with a state of mistrust, and thus they need to be analyzed further using the standard deviation. By comparing the differences in alpha waves under a state of trust when the baseline is zero, $96.42 \%$ of the participants showed an increase in trust, with a standard deviation of 1.13E08E (Figure 6). By comparing the differences in the beta waves under a state of trust when the baseline is zero, $100 \%$ of participants showed an increase in trust, with a standard deviation of 1.18E-08E (Figure 6(b)). By comparing the differences in the gamma waves under a state of mistrust when the baseline was zero, $96.42 \%$ of the participants showed an increase in mistrust, with a standard deviation of 4.08E-10E (Figure 6(c)). According to the comparisons of the differences between trust and mistrust, the alpha and beta waves increased under a state of trust, whereas the gamma waves increased under a state of mistrust.

\section{Conclusions}

In this study, human trust in automation was investigated using EEG signals to identify specific brainwaves under states of trust and mistrust. According to the results of the experiment, the alpha and beta waves were stronger under a state of trust, whereas the gamma waves were stronger under a state of mistrust (see Figures 4 and 5). This section investigates neurological relationships grounded based on neuroscience that are critical for understanding the relationship of alpha, beta, and gamma waves to trust. Alpha waves are related to meditation [16] and reducing stress and anxiety [17]. Beta waves are associated with conscious activities and can be used as guidelines for measuring the cognitive process [18]. Beta activity is important because it can enhance the levels of concentration, attention, emotional stability, and energy [19]. As the fastest brainwaves, gamma waves can indicate anxiety and advanced cognitive information, such as reasoning and judgment [20].
By understanding the brain waves, alpha and beta waves under states of trust are related to the normal cognitive process. However, gamma waves under states of mistrust are related to complex cognitive processes caused by increased stress and anxiety.

Previous studies [21, 22] have used EEG signals to deal with such factors as risk and feedback, which affect human trust and decision-making, and support the results of this research. Using EEG signals recorded through a competitive decision-making game, Cohen [23] found that increased feedback processing lowers the power delta and theta waves, and increases the power of alpha and beta waves.

The findings from the present study will contribute to the use of neurological technologies to measure the level of trust in automation of a human operator, which may affect the decisionmaking and overall performance of automation in different industries. This research can be valuable in the design of automated systems for the development of a user-friendly interface, as well as effective training, thereby increasing the trust of the human operator and decreasing the workload. Further, this research can be applied to monitor the psychological state of human operators during complex automation, such as pilots flying automated aircrafts or captains navigating automated ships.

\section{Related Studies}

Previous studies have been conducted on trust in automation using the power spectrum or PSD as a reliable and fast method for analyzing brain waves. Wang et al. [24] investigated the neural activities of human trust in automation using EEG signals and analyzed the brain signals using the PSD and a Fourier analysis. The authors applied an investment game with different levels of artificial agents and identified specific brain regions, namely, the frontal and occipital areas, as related to trust in automation. However, the present study used a simulated driving game with manual or automatic control and identified specific brain waves, namely, alpha and beta waves for the state of trust and gamma waves for the state of mistrust. Seet et al. [25] investigated how trust is affected by autonomous vehicle malfunction using EEG signals determined through an experiment using conditionally automated and fully automated driving modes. Although they showed that trust is affected not directly by malfunctions in automation but by the inability of a human operator to reduce the risk from such malfunctions, the results of the present study demonstrate that a low performance of automatic control affects the level of trust. Trust can be affected by various factors such 


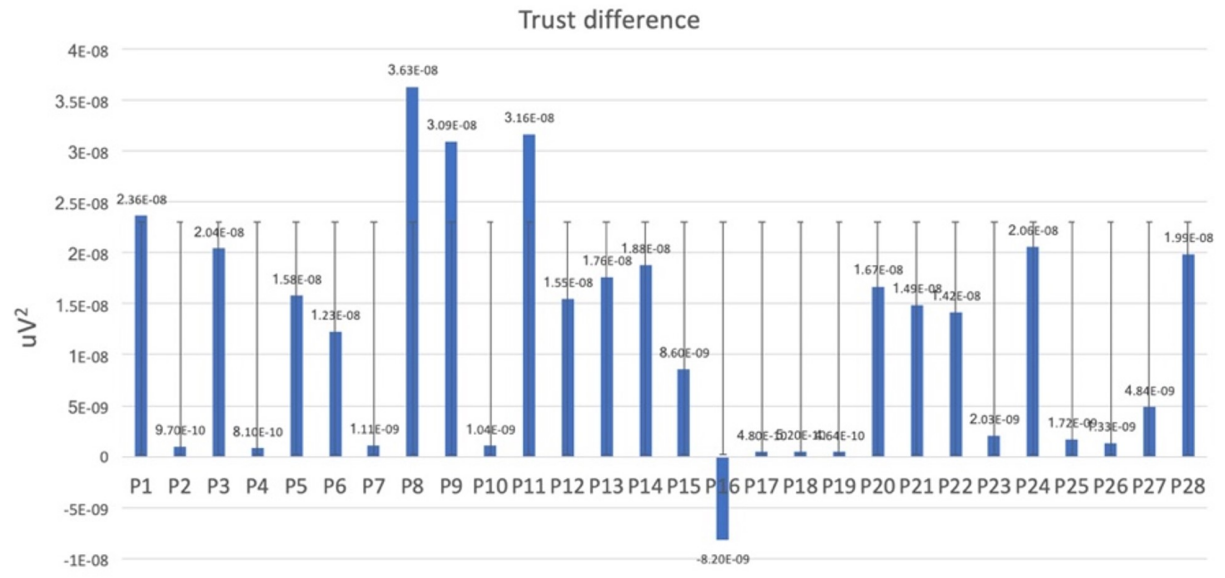

(a)

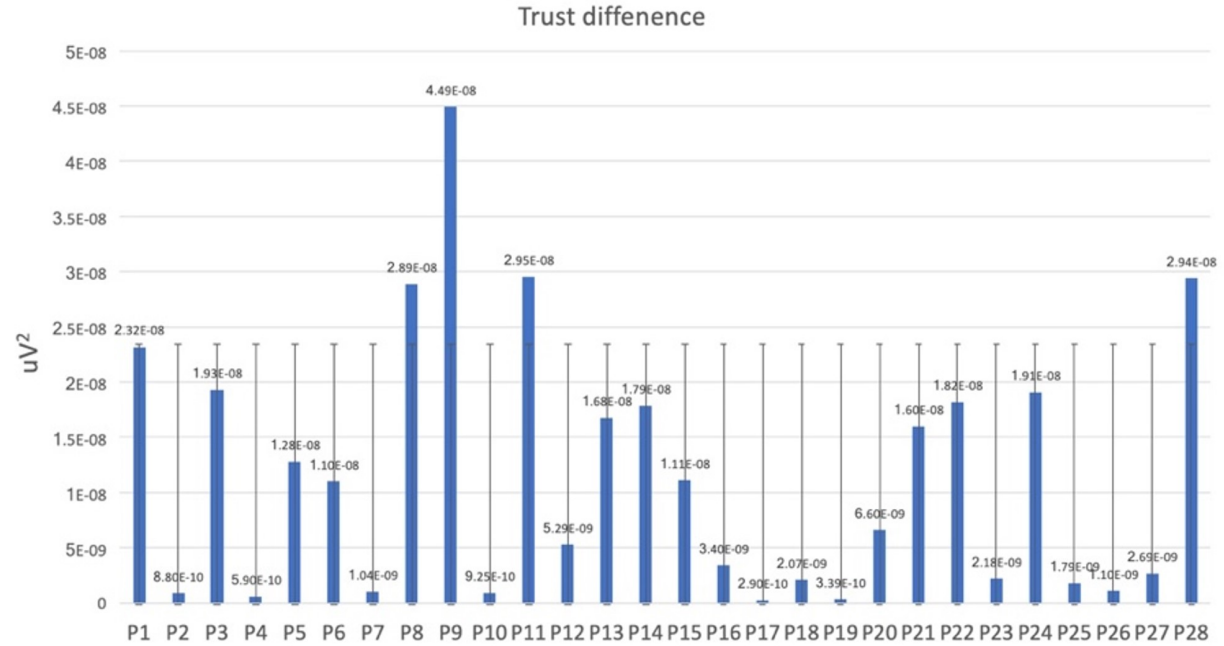

(b)

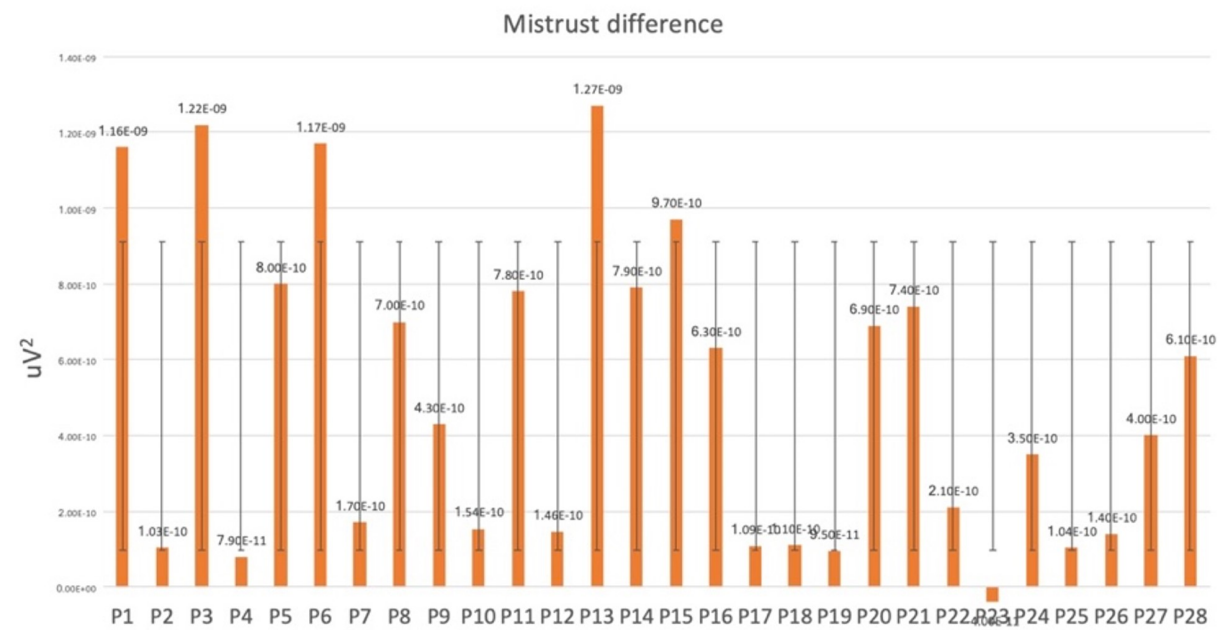

(c)

Figure 6. (a) Alpha waves of participants $(\mathrm{N}=28)$, (b) beta waves of participants $(\mathrm{N}=28)$, and (c) gamma waves of participants (N=28), under states of trust and mistrust, respectively. 


\section{Appendix}

\section{Scales for Trust between People and Automation}

Below is a survey to evaluate the level of trust between people and automation for each trial.

There are scales for you to rate the level of trust of the automatic system when you choose automatic or manual control before operation. Please check the level of trust for each trial.

Record sheet of your decision for each trial (Note: $\mathrm{A}=$ Automatic control, $\mathrm{M}=$ Manual control)

\begin{tabular}{|l|l|l|l|l|l|l|l|l|l|l|}
\hline & Trial 1 & Trial 2 & Trial 3 & Trial 4 & Trial 5 & Trial 6 & Trial 7 & Trial 8 & Trial 9 & Trial 10 \\
\hline A or M & & & & & & & & & & \\
\hline
\end{tabular}

Question: I can trust the automatic system (Note: not at all $=1$, extremely $=7$ )

\begin{tabular}{|l|l|l|l|l|l|l|l|}
\hline & 1 & 2 & 3 & 4 & 5 & 6 & 7 \\
\hline Trial 1 & & & & & & & \\
\hline
\end{tabular}

\begin{tabular}{|c|c|c|c|c|c|c|c|}
\hline & 1 & 2 & 3 & 4 & 5 & 6 & 7 \\
\hline Trial 2 & & & & & & & \\
\hline
\end{tabular}

\begin{tabular}{|c|c|c|c|c|c|c|c|}
\hline & 1 & 2 & 3 & 4 & 5 & 6 & 7 \\
\hline Trial 3 & & & & & & & \\
\hline
\end{tabular}

\begin{tabular}{|c|c|c|c|c|c|c|c|}
\hline & 1 & 2 & 3 & 4 & 5 & 6 & 7 \\
\hline Trial 4 & & & & & & & \\
\hline
\end{tabular}

\begin{tabular}{|c|c|c|c|c|c|c|c|}
\hline & 1 & 2 & 3 & 4 & 5 & 6 & 7 \\
\hline Trial 5 & & & & & & & \\
\hline
\end{tabular}

\begin{tabular}{|c|c|c|c|c|c|c|c|}
\hline & 1 & 2 & 3 & 4 & 5 & 6 & 7 \\
\hline Trial 6 & & & & & & & \\
\hline
\end{tabular}

\begin{tabular}{|c|c|c|c|c|c|c|c|}
\hline & 1 & 2 & 3 & 4 & 5 & 6 & 7 \\
\hline Trial 7 & & & & & & & \\
\hline
\end{tabular}

\begin{tabular}{|l|l|l|l|l|l|l|l|}
\hline & 1 & 2 & 3 & 4 & 5 & 6 & 7 \\
\hline Trial 8 & & & & & & & \\
\hline
\end{tabular}

\begin{tabular}{|l|l|l|l|l|l|l|l|}
\hline & 1 & 2 & 3 & 4 & 5 & 6 & 7 \\
\hline Trial 9 & & & & & & & \\
\hline
\end{tabular}

\begin{tabular}{|l|l|l|l|l|l|l|l|}
\hline & 1 & 2 & 3 & 4 & 5 & 6 & 7 \\
\hline Trial 10 & & & & & & & \\
\hline
\end{tabular}

as motivation, expectation, or predictability, and is influenced by various factors, such as motives, intentions, and actions [26]. It is therefore necessary to research these factors further. Akash et al. [27] developed trust-sensor models, in which machines can detect psychophysiological features of trust in humans using EEG signals and the galvanic skin response. Whereas they examined how intelligent machines can detect trust in humans, the present paper examined how human trust in automation can be measured. Interactions between humans and machines are increasing in number, and in-depth research on trust between humans and machines is therefore needed.

\section{Conflict of Interest}

No potential conflict of interest relevant to this article was reported. 


\section{References}

[1] J. D. Lee, and K. A. See, "Trust in technology: designing for appropriate reliance," Human Factors, vol. 46, no. 1, pp. 50-80, 2004. https://doi.org/10.1518\%2Fhfes.46.1. 50_30392

[2] B. M. Muir, "Trust between humans and machines, and the design of decision aids," International Journal of ManMachine Studies, vol. 27, no. 5-6, pp. 527-539, 1987. https://doi.org/10.1016/S0020-7373(87)80013-5

[3] A. M. Bisantz and Y. Seong, "Assessment of operator trust in and utilization of automated decision-aids under different framing conditions," International Journal of Industrial Ergonomics, vol. 28, no. 2, pp. 85-97, 2001. https://doi.org/10.1016/S0169-8141(01)00015-4

[4] L. Wang, R. Law, K. Hung, and B. D. Guillet, "Consumer trust in tourism and hospitality: a review of the literature," Journal of Hospitality and Tourism Management, vol. 21, pp. 1-9, 2014. https://doi.org/10.1016/j.jhtm.2014.01.001

[5] X. Cheng, S. Fu, and G. J. de Vreede, "Understanding trust influencing factors in social media communication: a qualitative study," International Journal of Information Management, vol. 37, no. 2, pp. 25-35, 2017. https://doi. org/10.1016/j.ijinfomgt.2016.11.009

[6] J. Y. Jian, A. M. Bisantz, and C. G. Drury, "Foundations for an empirically determined scale of trust in automated systems," International Journal of Cognitive Ergonomics, vol. 4, no. 1, pp. 53-71, 2000. https://doi.org/10.1207/ S15327566IJCE0401_04

[7] J. D. Lee and N. Moray, "Trust, self-confidence, and operators' adaptation to automation," International Journal of Human-Computer Studies, vol. 40, no. 1, pp. 153-184, 1994. https://doi.org/10.1006/ijhc.1994.1007

[8] A. Dimoka, "Brain mapping of psychological processes with psychometric scales: an fMRI method for social neuroscience," NeuroImage, vol. 54, pp. S263-S271, 2011. https://doi.org/10.1016/j.neuroimage.2010.05.007

[9] L. A. Casado-Aranda, A. Dimoka, and J. SanchezFernandez, "Consumer processing of online trust signals: a neuroimaging study," Journal of Interactive Marketing, vol. 47, pp. 159-180, 2019. https://doi.org/10.1016/j. intmar.2019.02.006
[10] L. M. Hirshfield, S. H. Hirshfield, S. Hincks, M. Russell, R. Ward, and T. Williams, "Trust in human-computer interactions as measured by frustration, surprise, and workload," in Foundations of Augmented Cognition. Heidelberg, Germany: Springer, 2011, pp. 507-516. https: //doi.org/10.1007/978-3-642-21852-1_58

[11] S. Y. Dong, B. K. Kim, K. Lee, and S. Y. Lee, "A preliminary study on human trust measurements by EEG for human-machine interactions," in Proceedings of the 3rd International Conference on Human-Agent Interaction, Daegu, South Korea, 2015, pp. 265-268. https: //doi.org/10.1145/2814940.2814993

[12] G. A. Light, L. E. Williams, F. Minow, J. Sprock, A. Rissling, R. Sharp, N. R. Swerdlow, and D. L. Braff, "Electroencephalography (EEG) and event-related potentials (ERPs) with human participants," Current Protocols in Neuroscience, vol. 52, no. 1, pp. 6-25, 2010. https://doi.org/10.1002/0471142301.ns0625s52

[13] G. H. Klem, H. O. Luders, H. H. Jasper, and C. Elger, "The ten-twenty electrode system of the International Federation. The International Federation of Clinical Neurophysiology," Electroencephalography and clinical neurophysiology Supplement, vol. 52, pp. 3-6, 1999.

[14] C. Kim, J. Sun, D. Liu, Q. Wang, and S. Paek, "An effective feature extraction method by power spectral density of EEG signal for 2-class motor imagery-based BCI," Medical \& Biological Engineering \& Computing, vol. 56, no. 9, pp. 1645-1658, 2018. https://doi.org/10.1007/s11517017-1761-4

[15] J. A. Meltzer, M. Negishi, L. C. Mayes, and R. T. Constable, "Individual differences in EEG theta and alpha dynamics during working memory correlate with fMRI responses across subjects," Clinical Neurophysiology, vol. 118, No. 11, pp. 2419-2436, 2007. https://doi.org/10.1016/ j.clinph.2007.07.023

[16] M. Prestel, R. Riedl, R. Stark, and U. Ott, "Enhancing mindfulness by combining neurofeedback with meditation," Journal of Consciousness Studies, vol. 26, no. 7-8, pp. 268-293, 2019.

[17] A. Rajendran, R. Sudeshraj, and S. Sureshkumar, "Phytonutrients: stress and relaxation dietary health food supplements," The Pharma Innovation Journal, vol. 8, no. 5, pp. 799-802, 2019. 
[18] H. F. Posada-Quintero, N. Reljin, J. Bolkhovsky, A. D. Orjuela-Canon, and K. Chon, "Brain activity correlates with cognitive performance deterioration during sleep deprivation," Frontiers in Neuroscience, vol. 13, article no. 1001, 2019. https://doi.org/10.3389/fnins.2019.01001

[19] J. Y. Arana-Llanes, G. Gonzalez-Serna, R. Pineda-Tapia, V. Olivares-Peregrino, J. J. Ricarte-Trives, and J. M. Latorre-Postigo, "EEG lecture on recommended activities for the induction of attention and concentration mental states on e-learning students," Journal of Intelligent \& Fuzzy Systems, vol. 34, no. 5, pp. 3359-3371, 2018. https://doi.org/10.3233/JIFS-169517

[20] R. Jobdas, A. Jani, and R. Vyas, "BCI in classroom, system for assessing dynamic state of cognitive performance using EEG signals," International Journal of Research in Engineering and Innovation, vol. 2, no. 2, pp. 195-200, 2018.

[21] K. Ota, M. Shinya, and K. Kudo, "Transcranial direct current stimulation over dorsolateral prefrontal cortex modulates risk-attitude in motor decision-making," Frontiers in Human Neuroscience, vol. 13, article no. 297, 2019. https://doi.org/10.3389/fnhum.2019.00297

[22] P. Sacre, M. S. Kerr, S. Subramanian, Z. Fitzgerald, K. Kahn, M. A. Johnson, et al., "Risk-taking bias in human decision-making is encoded via a right-left brain push-pull system," Proceedings of the National Academy of Sciences, vol. 116, no. 4, pp. 1404-1413, 2019. https: //doi.org/10.1073/pnas.1811259115

[23] M. X. Cohen, C. E. Elger, and J. Fell, "Oscillatory activity and phase-amplitude coupling in the human medial frontal cortex during decision making," Journal of Cognitive Neuroscience, vol. 21, no. 2, pp. 390-402, 2008. https://doi.org/10.1162/jocn.2008.21020

[24] M. Wang, A. Hussein, R. F. Rojas, K. Shafi, and H.A. Abbass, "EEG-based neural correlates of trust in humanautonomy interaction," in Proceedings of 2018 IEEE Symposium Series on Computational Intelligence (SSCI), Bangalore, India, 2018, pp. 350-357. https://doi.org/10.1109/ SSCI.2018.8628649

[25] M. Seet, J. Harvy, R. Bose, A. Dragomir, A. Bezerianos, and N. Thakor, "Differential impact of autonomous vehicle malfunctions on human trust," IEEE Transactions on Intelligent Transportation Systems, 2020. https: //doi.org/10.1109/TITS.2020.3013278

[26] R. Bhattacharya, T. M. Devinney, and M. M. Pillutla, "A formal model of trust based on outcomes," Academy of Management Review, vol. 23, no. 3, pp. 459-472, 1998. https://doi.org/10.5465/amr.1998.926621

[27] K. Akash, W. L. Hu, N. Jain, and T. Reid, "A classification model for sensing human trust in machines using EEG and GSR," ACM Transactions on Interactive Intelligent Systems, vol. 8, no. 4, article no. 27, 2018. https://doi.org/ $10.1145 / 3132743$

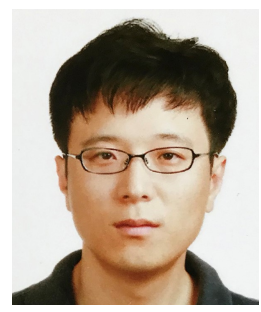

Seeung $\mathrm{Oh}$ is an adjunct professor in the department of Applied Engineering Technology at North Carolina Agricultural and Technical State University, Greensboro, USA. He earned $\mathrm{PhD}$ in Industrial and Systems Engineering at North Carolina Agricultural and Technical State University in 2018. Dr. Oh's research interests include human factors and ergonomics design with neurological technologies, human trust in automated systems, decision-making and brain-computer interface (BCI) and user interface(UI) design and information visualization.

E-mail: soh1@ncat.edu

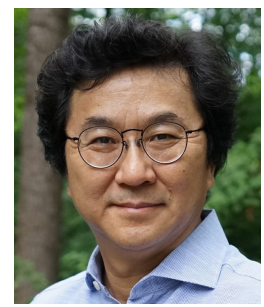

Younho Seong received the B.Sc. and M.Sc. degree in industrial engineering from Inha University, South Korea, in 1991 and 1993 respectively, and Ph.D. degree in industrial engineering from State University of New York at Buffalo in 2002. $\mathrm{He}$ is currently Professor of industrial and systems engineering with North Carolina A\&T State University, Greensboro, NC, USA. His research interests include cognitive engineering, ecological approaches to human judgment $\&$ decision-making, machine learning, human trust in automation, social aspect of autonomous vehicles, human-robot interaction, designing interventions to support human cognition, virtual and augmented reality, neuroergonomics, and brain-computer interface.

E-mail: yseong@ncat.edu 


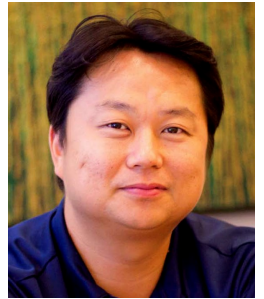

Sun $\mathbf{Y i}$ is an associate professor of Mechanical Engineering at North Carolina A\&T State University. He has developed new and novel methods for sensing and control algorithms for dynamic systems, which are adaptive and robust. The methods have also been applied to networked robots and UAVs/UGVs using AI, neural networks, sensor fusion, machine visions and adaptive control. He has managed research projects supported by DoD, NASA, Dept. Energy, and Dept. Transportation.

E-mail: syi@ncat.edu

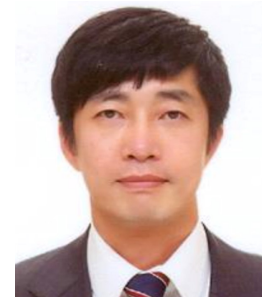

Sangsung Park was born in Korea. He received his Master and $\mathrm{PhD}$ of Engineering degree in industrial engineering from Korea University. He was research and assistant Professor at Korea University from 2006 to 2018. He is an assistant Professor at the CheongJu University. His research interests are Patent Analysis, Data Mining, Management of Technology, and Technology Evaluation.

E-mail: hanyul@cju.ac.kr 